\title{
Two cases of black human breast milk not related to minocycline. A sphingolipidomic approach
}

\author{
Laura Cerquiglini ${ }^{1}$, Stefania Troiani ${ }^{1}$, Chiara Gizzi ${ }^{1}$, Michele Dei Cas ${ }^{2}$, Rita Paroni $^{2}$, Paola Signorelli ${ }^{2}$, Antonella \\ Mencacci $^{3}$, Maurizio Radicioni ${ }^{1}$, Stefano Pasquino ${ }^{4}$, Maria Cristina De Lio ${ }^{5}$, Lina Cossignani ${ }^{6}$, Giuseppa \\ Verducci $^{6}$, Carmela Conte ${ }^{6}$, Tommaso Beccari ${ }^{6}$, Samuela Cataldi, ${ }^{6}$ Elisabetta Albi ${ }^{6 *}$
}

${ }^{1}$ Struttura Complessa di Neonatologia e Terapia Intensiva Neonatale, Azienda Ospedaliera di Perugia - Santa Maria della Misericordia, Perugia, Italy; ${ }^{2}$ Department of Health Sciences, Università degli Studi di Milano, Milan, Italy; ${ }^{3}$ Medical Microbiology, Department of Medicine and Surgery, University of Perugia, Perugia, Italy; ${ }^{4}$ Department of Cardiac Surgery, Azienda Ospedaliera di Perugia - Santa Maria della Misericordia, Perugia, Italy; ${ }^{5}$ Igiene e Organizzazione dei Servizi Ospedalieri, Azienda Ospedaliera di Perugia - Santa Maria della Misericordia, Perugia, Italy; ${ }^{6}$ Department of Pharmaceutical Sciences, University of Perugia, Perugia, Italy

*Corresponding Author: Elisabetta Albi, Department of Pharmaceutical Sciences, University of Perugia, Perugia 06126, Italy. Email: elisabetta.albi@unipg.it

Received: 19 January 2022; Accepted: 13 February 2022; Published: 4 March 2022

(C) 2022 Codon Publications

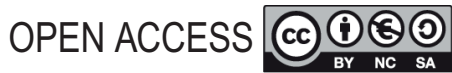

OPINION PAPER

\begin{abstract}
Different colors of human breast milk (HBM) are reported in literature, and black milk is produced only during minocycline therapy. We herein report two cases of black/dark gray color HBM without minocycline involvement. We analyzed both milk samples and compared the data with two control HBM samples taken from two mothers who had the same dietary behaviors and took the same supplements (iron) as done by the mothers under study. Results indicated that the black color was not due to iron intake, disease or infection. With Sudan III stain, specific for lipids, dark precipitates were evident. Antioxidant power was much higher in studied milk samples than in control samples. As antioxidants at high levels become pro-oxidants, our data suggested possible lipid oxidation. Sphingolipid profile of black milk samples demonstrated accumulation of sphingomyelin and ceramide, which could be a sign of impaired lipid metabolism. It was concluded that iron supplementation was not responsible for HBM pigmentation, but altered biochemical mechanisms in the mammary gland could be implicated. In our experience, dark color HBM did not represent an absolute indication for discontinuation of breastfeeding.
\end{abstract}

Keywords: black milk, breast milk, ceramide, fatty acids, human milk, sphingomyelin

\section{Introduction}

Human breast milk (HBM) is universally recognized as the preferred source of neonatal nutrition. It is an extremely complex biological fluid. Some components vary with the maternal diet, while others are independent of this and depend on metabolic activity of the mammary gland (Dei Cas et al., 2020). Thus, breast milk is species-specific and reflects the requirement of infants (Tripaldi et al., 2021). In fact, HBM protects infants from diseases as they wait for their immune systems to mature (Hanson and Korotkova, 2002), from dysfunctional lipid metabolism and gut dysbiosis (Norris et al., 2019). Moreover, HBM has good antioxidant properties (Codini et al., 2020) and it is essential for the cognitive maturation (Skinner and Narchi, 2021).

Throughout lactation, HBM undergoes a gradual biochemical modification in lipid and protein concentration so that the milk is commonly classified into colostrum, 
transitional milk and mature milk (Jenness, 1979). Colostrum, the first milk produced after parturition, is rich in proteins and growth factors and has high concentrations of secretory immunoglobulin, thus indicating its immunological function (Castellote et al., 2011). Transitional milk is produced around the third-fifth day after childbirth until 20 days-1 month of child birth. After this, mature milk is produced and it lasts for the duration of breastfeeding. Transitional milk has high contents of fat and carbohydrates and is low in minerals and proteins. Mature milk is truly abundant in fats and carbohydrates and has an optimal protein and minerals intake in relation to the increased nutritional needs of the newborn (Riordan and Wambach, 2014).

The color of HBM changes with lactation. If sometimes, the colostrum appears clear, thin and watery, at other times it is often yellow or orange, thanks to the presence of high levels of beta-carotene (Patton et al., 1990). Transitional milk typically changes from yellow to white, and mature milk is first clear or bluish and afterwards white or yellow in color (Riordan and Wambach, 2014).

Milk color may vary physiologically depending on diet, nutritional supplements and medications. HBM can appear pink, red and orange with fruit drinks or green with green color beverages, green vegetables, vitamins or mineral supplements in diet (Yazgan et al., 2012) or intake of blue-green algae (Naor et al., 2019). However, the color of HBM may change for different reasons, as pink color is described in colonization of Serratia marcescens (Ayuzo del Valle and Treviño Salinas, 2014; Jones et al., 2014; Quinn et al., 2018). Moreover, green color is reported after administration of propofol (Birkholz et al., 2009; Rainone et al., 2018). However, black HBM is reported only in relation to minocycline therapy (Basler, et al., 1985; Hunt et al., 1996; Lawrence, 1985).

Although different observations have been reported on the colors of HBM, possible modifications in lipid components have not been studied yet. Since the sphingomyelin (SM) present in breast milk is important for the maturation of child's nervous system (Jiang et al., 2021), we particularly focused on sphingolipidomic study.

We report two cases of black HBM produced during early lactation by two mothers not under antibiotic therapy but both taking iron for anemia. Iron intake and breastfeeding were discontinued. Milk color in both cases turned white within a few days of iron discontinuation and mothers restarted breastfeeding without any incident. As a control (CRT), two clear/white milk samples from two mothers on iron supplementation were examined. We investigated and reported microbiological, cytological and biochemical aspects of both samples.

\section{Materials and Methods}

\section{Population and sample collection procedure}

BLUD in Perugia, Italy (Banca del Latte Umano Donato, Struttura Complessa di Neonatologia e Terapia Intensiva Neonatale-Azienda Ospedaliera Santa Maria della Misericordia-Perugia, Italy) provided human milk samples. Two black milk samples under investigation and two CRT white milk samples collected from healthy iron-supplemented mothers were examined for the study. All procedures were performed according to the indications of Bioethics Committee, and the women signed informed consent. Mothers were invited to answer questions about: (1) age; (2) cigarette use; (3) use of medical products containing nicotine; (4) alcohol use; (5) use of restrictive or specific diets; and (6) medical therapy. Immediately after harvesting, milk sample was kept for microbiological analysis and the remaining amount was submitted for Holder pasteurization (HoP) by heating it to $62.5^{\circ} \mathrm{C}\left(145^{\circ} \mathrm{F}\right)$ for $30 \mathrm{~min}$, and then cooling it back to $4-10^{\circ} \mathrm{C}$; it is a globally used method to ensure distribution of microbiologically safe milk to infants (Codini et al., 2020). After pasteurization, microbiological analysis was performed again and the milk samples were stored at $-20^{\circ} \mathrm{C}$ in a freezer before analysis.

\section{Materials}

Anhydrous sodium sulfate, chloroform, hexane, methanol and potassium hydroxide were purchased from Carlo Erba Reagents (Milan, Italy). Sudan III, codex S-4131, was obtained from Sigma Chemical Co. (St. Louis, MO, USA). Supelco ${ }^{\text {tm }}$ 37-component fatty acid methyl esters (FAME) mix, containing methyl esters of 37 fatty acids, was supplied by Supelco (Bellefonte, PA, USA). Lipids standards were purchased from Avanti Polar Lipids (Alabaster, AL, USA). Chemicals, all analytical grade, were purchased from Sigma-Aldrich (St. Louis, MO, USA). All aqueous solutions were prepared using purified water of Milli-Q grade (Burlington, MA, USA).

\section{Microbiological analysis}

Milk samples were collected aseptically by using breast pump and an aliquot was taken for Gram staining and culture. Quantitative culture was performed onto chocolate, blood supplemented with colistin and nalidixic acid, McConkey, mannitol salt and Sabouraud agar plates (all media were acquired from Becton Dickinson, East Rutherford, NJ, USA). Plates were incubated in $\mathrm{CO}_{2}$ or air-incubators and were read for development of colonies after being kept overnight and 48-h incubation. 
Microbial load in milk samples was measured as colony forming units $/ \mathrm{mL}$. Colonies were identified using the Bruker MALDI Biotyper instrument (Bruker Daltonik $\mathrm{GmbH}$, Bremen, Germany), and antimicrobial susceptibility testing was performed with the BD Phoenix automatic system (Becton Dickinson).

\section{Sudan III staining}

Saturated Sudan III was prepared by diluting 0.5-g powder in $100-\mathrm{mL} 99 \%$ isopropanol. The solution was allowed to set for 2 days before using the supernatant. Working Sudan III stain was obtained by diluting $6 \mathrm{~mL}$ of saturated Sudan III dye in $4 \mathrm{~mL}$ of distilled water, and letting it stand for $10 \mathrm{~min}$ and filtering the solution before use. Each sample, $50 \mu \mathrm{L}$, was dispersed on polarized slides and allowed to air-dry for $24 \mathrm{~h}$. The samples were stained with working Sudan III, left at room temperature for $10 \mathrm{~min}$, washed with distilled water, and allowed to air-dry. The stained slides were examined with Euromex-Holland FE 2915 microscope (BD Arnhem, The Netherlands) equipped with a DC5000 CMEX-5.0 pixel digital USB camera system and analyzed at $40 \times$ magnification.

\section{Antioxidant assay by Oxygen Radical Absorbance Capacity (ORAC)}

The antioxidant capacity of human milk samples was determined using the ORAC method as reported by Ceccarini et al. (2016). We used ORAC method because it is a robust and reliable method. In fact, the ORAC assay, with other common measures of antioxidant capacity, including ferric ion-reducing antioxidant power and trolox equivalence antioxidant capacity assays, is regarded as a preferable method because of its biological relevance (Codini et al., 2015). A duplicate extraction was performed for each sample and used to evaluate lipophilic ORACFL (L-ORACFL) and hydrophilic ORACFL (H-ORACFL) values. Evaluations of lipophilic and hydrophilic ORACFL values in the samples were performed separately, and the total antioxidant capacity was calculated by adding L-ORACFL and H-ORACFL values. ORACFL assays were conducted on a FLUOstar OPTIMA microplate fluorescence reader (BMG Labtech, Offenburg, Germany) at an excitation wavelength of $485 \mathrm{~nm}$ and an emission wavelength of $520 \mathrm{~nm}$. 2,20-Azobis (2-methylpropionamide) dihydrochloride was used as a peroxyl radical generator; trolox was used as a reference antioxidant standard; and fluorescein was used as a fluorescent probe. The data were expressed as micromoles of trolox equivalents (TE) per gram of sample $(\mu \mathrm{mol} \mathrm{TE} / \mathrm{g})$.

\section{Gas chromatographic analysis of fatty acids}

Milk lipid fraction was extracted using a chloroformmethanol mixture $(2: 1, \mathrm{v} / \mathrm{v})$, following the procedure reported previously (Codini et al., 2020). The FAME of total lipids was prepared by transmethylation with methanolic $\mathrm{KOH}$ and analyzed using high-resolution gas chromatography. A DANI 1000DPC gas-chromatograph (Norwalk, CT, USA), equipped with a split-splitless injector and a flame ionization detector, was used. The FAME separation was performed with a CP-select CB for FAMEfused silica capillary column $(50 \mathrm{~m} \times 0.25 \mathrm{~mm}$ I.D., 0.25$\mu \mathrm{m}$ F.T.; Varian, Superchrom, Milan, Italy). The injector and detector temperatures were $250^{\circ} \mathrm{C}$. The oven temperature was $60^{\circ} \mathrm{C}$, held for $5 \mathrm{~min}$, then raised to $225^{\circ} \mathrm{C}$ at a rate of $3^{\circ} \mathrm{C} / \mathrm{min}$; the final temperature was held for $10 \mathrm{~min}$. The chromatograms were acquired and processed using the Clarity Integration software (DataApex Ltd., Prague, Czech Republic). A standard solution containing 37 FAME was used to identify individual fatty acids. High resolution gas chromatography analysis was conducted in triplicate.

\section{Liquid chromatography with tandem mass spectrometry}

Sphingolipid (Sph) extraction and liquid chromatography coupled with tandem mass spectrometry (LC-MS/MS) analyses were performed as described by Dei Cas et al. (2020). Sphingolipids were extracted from three independent $25-\mu \mathrm{L}$ aliquots of HBM using monophasic solvent extraction (chloroform:methanol:water-30:60:10, v/v/v). These were analyzed with liquid chromatography (Dionex 3000 UltiMate, ThermoFisher Scientific, Waltham, MA, USA) coupled with tandem mass spectrometer (AB Sciex 3200 QTRAP, Sciex, Vaughn, ON, Canada). The separation was achieved by a reversed-phase analytical column (Acquity BEH C8 $100 \times 2.1 \mathrm{~mm} \times 1.7 \mu \mathrm{m}$; Waters, Milford, MA, USA) through a linear gradient between eluent A ( $0.2 \%$ formic acid, 2-mM ammonium formate in water solution) and eluent B ( $0.2 \%$ formic acid, $1-\mathrm{mM}$ ammonium formate in methanol). Quantitative analysis was performed interpolating each peak area of analyte/ area internal standards with a calibration curve for each sphingolipid.

\section{Statistical analysis}

Statistical analysis of lipidomic study was performed with GraphPad Prism 7.0 (GraphPad Software Inc, La Jolla, CA, USA). Statistical differences of fatty acids and sphingolipidomic study were investigated by unpaired $t$-test. Graphs were represented as mean $\pm \mathrm{SD}$, and statistical significance was set as $p<0.05$. 


\section{Results}

\section{Mothers}

Two women, A: 26-year-old, and B: 43-year-old, were examined for the study. The women were enrolled because of the dark color of their colostrum. It emerged from the analysis of their questionnaires that both mothers neither smoked nor used alcohol and followed a Mediterranean diet without restrictions. Mother A was under iron supplementation due to physiological anemic condition during pregnancy. Mother B took iron for anemia as well and methyldopa for hypertension arising during pregnancy. Moreover, she supplemented the diet with folic acid and docosahexaenoic acid (DHA). In order to address possible bias because of the suspected role of iron, we also included in the study two healthy mothers, aged 26 and 43 years, taking iron but producing light color milk, as CRT samples. The selected mothers were neither affected by any chronic pathology nor had received any recent transfusion; they were only anemic and followed a Mediterranean diet.

\section{Characteristics of human breast black milk samples}

Microbiology was aspecific. Milk samples of control as well as A and B mothers were positive to culture test for bacteria but negative to culture test for yeasts. Organisms identified in the milk of control 1 mother were Staphylococcus epidermidis (20.000 colony-forming units $[\mathrm{CFU}] / \mathrm{mL})$, Acinetobacter junii (>100.000 CFU/mL), Acinetobacter ursingii (3.000 CFU/ $\mathrm{mL})$ and Staphylococcus aureus $(<3.000 \mathrm{CFU} / \mathrm{mL})$. Organisms identified in the milk of control 2 mother were Staphylococcus epidermidis (10.000 CFU/mL) and Staphylococcus haemolyticus (1.000 CFU/mL). Organisms identified in the milk sample of mother A were Staphylococcus lugdunenesis (20.000 CFU/mL) and Staphylococcus aureus (5.000 CFU/mL). Organisms identified in the milk sample of mother B were Staphylococcus epidermidis (10.000 CFU/mL), Staphylococcus lugdunensis (800 CFU/mL) and Pseudomonas fluorescens (800 $\mathrm{CFU} / \mathrm{mL}$ ). After pasteurization, all microbiological tests were negative in all milk samples and the black color of milk samples A and B remained unchanged. Therefore, we decided to investigate the lipid component of black milk samples and compared them with the light milk samples. First, we performed milk staining with Sudan III. Figure 1 shows the presence of fat globules of different sizes in the two CRT samples with physiological orange color. In the two samples of black milk, black precipitations appeared in the central or peripheral part of fat globules.

The analysis of antioxidant capacity demonstrated that samples A and B had very high antioxidant capacity (Figure 2). ORAC value in CRT samples was
(A)

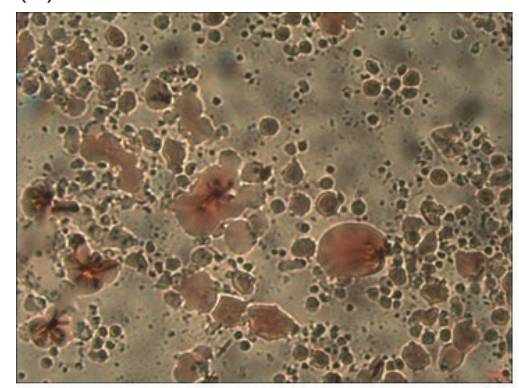

CTR1

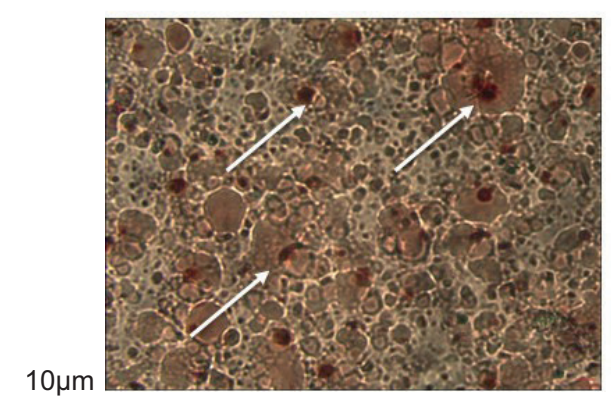

(B)

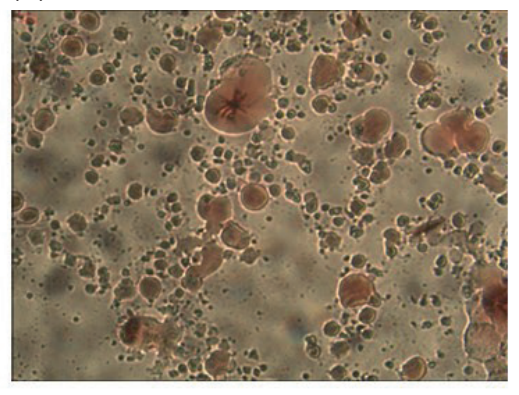

CTR2

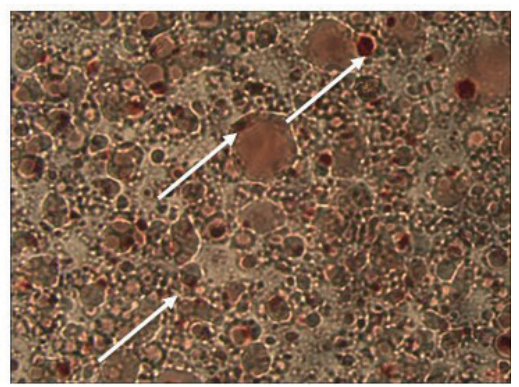

Figure 1. Image of milk samples stained with Sudan III. The arrows indicate black precipitates in fatty granules. Magnification 20x. 
$632.97+19.24 \mu \mathrm{molTE} / \mathrm{mL}$. In black milk sample $\mathrm{A}$, the value was $1826.09+46.95 \mu \mathrm{molTE} / \mathrm{mL}$ and in black milk sample B, it was $122.49+37.34 \mu \mathrm{molTE} / \mathrm{mL}$.

\section{Lipid composition of human breast black milk samples}

First, we measured fatty acids (FA) in HBM. Results of black milk samples A and B were compared with those of white milk CRT samples. We analyzed the general similarity and difference of total saturated fatty acids (sFA) and unsaturated fatty acids (uFA). Black milk sample A contained more uFA but black milk sample B had less sFA than CTR milk samples (Figure 3A). Interestingly, the sFA:uFA ratio was similar in black milk samples A and $B$ and it was lower than that of CRT milk samples (Figure 3B).

If each species of FA was examined, it became evident that in both black milk samples A and B, C16:1n-9, C18:3n-3, C20:1, C20:4, C22:5 and C22:6 were higher

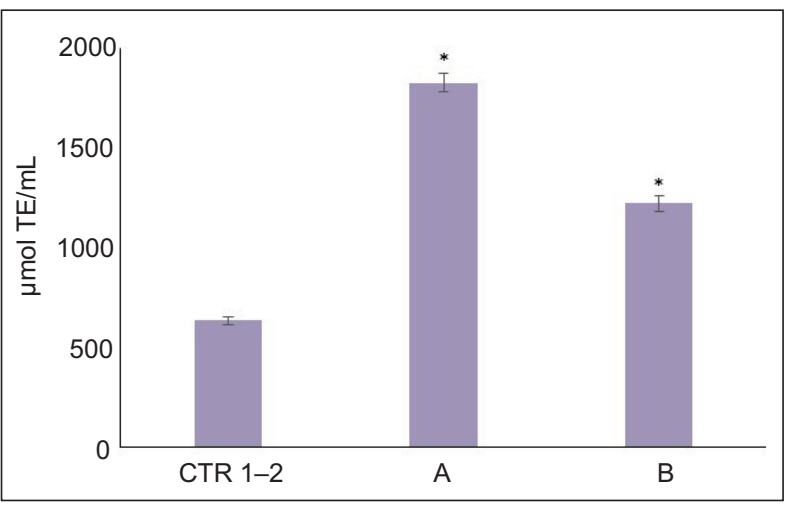

Figure 2. Antioxidant potential of human breast milk. CRT: control milk sample; $A$ and B: black milk samples. Data are expressed as mean \pm SD calculated as reported in 'Statistical analysis'. Significance of A and B versus CTR 1-2; * $p<0.05$. than in CRT milk samples (Figure 4). Moreover, milk sample A was particularly rich in C12:0 and C18:1n-9 content whereas milk sample B was deficient in C14:0 and $\mathrm{C} 16: 0$ contents (Figure 4).

We investigated possible changes in the composition of sphingolipids in black milk samples in comparison to CTR milk samples. The results highlight a higher level of sphingomyelin content in both black milk samples A and $\mathrm{B}$ (Figure 5A) but a higher level of ceramide (Cer) content in black milk sample A (Figure 5B).

In Figure 6, each sphingolipid species was reported. Of note, all sphingomyelin species were increased in milk samples A and B except 24:0 SM compared to CRT milk samples. 14:0 Cer, 16:0 Cer, 18:0 Cer, 18:1 Cer and 20:0 Cer were increased in milk sample A but only 10:0 Cer was enhanced in milk sample B. Dihydroceramide (DhCer) species was present only in milk sample A.

\section{Discussion}

Previous studies have demonstrated that color of HBM may vary physiologically, in relation to diet, nutritional supplements, infections and medications, from pink to orange, red and green (Ayuzo del Valle and Treviño Salinas, 2014; Jones et al., 2014; Naor et al., 2019; Quinn et al., 2018; Yazgan et al., 2012). The black color of HBM is reported only during minocycline treatment (Basler and Lynch, 1985; Hunt et al., 1996; Lawrence, 1985). However, here we report for the first time the case of two mothers who neither suffered from any pathology nor had minocycline therapy but had a black/deep dark color HBM. The only clinical aspect they had in common was iron supplementation. Mothers who had dark color milk as well as control mothers who had white color normal milk were using iron for anemia, suggesting that mineral intake was not responsible for milk's color, and
(A)

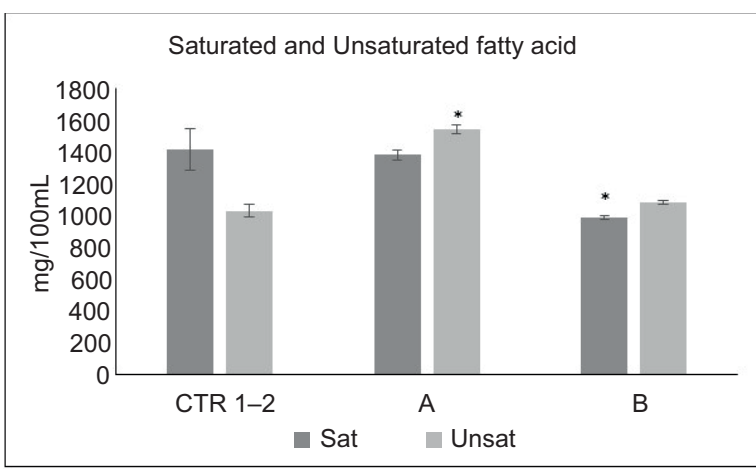

(B)

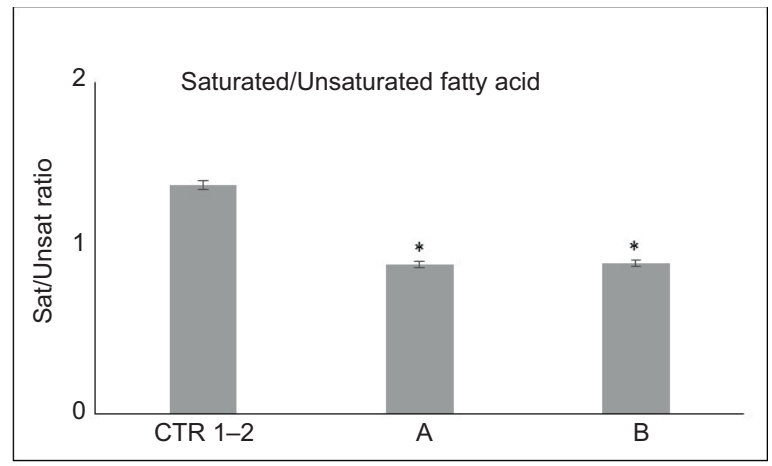

Figure 3. Composition of fatty acids in human breast milk. (A) Total saturated and total unsaturated fatty acids. (B) Ratio of saturated and unsaturated fatty acids. CRT: control milk samples; $A$ and B: black milk samples. Data are expressed as mean \pm SD calculated as reported in 'Statistical analysis'. Significance of A and B versus CTR 1-2; * $p<0.05$. 


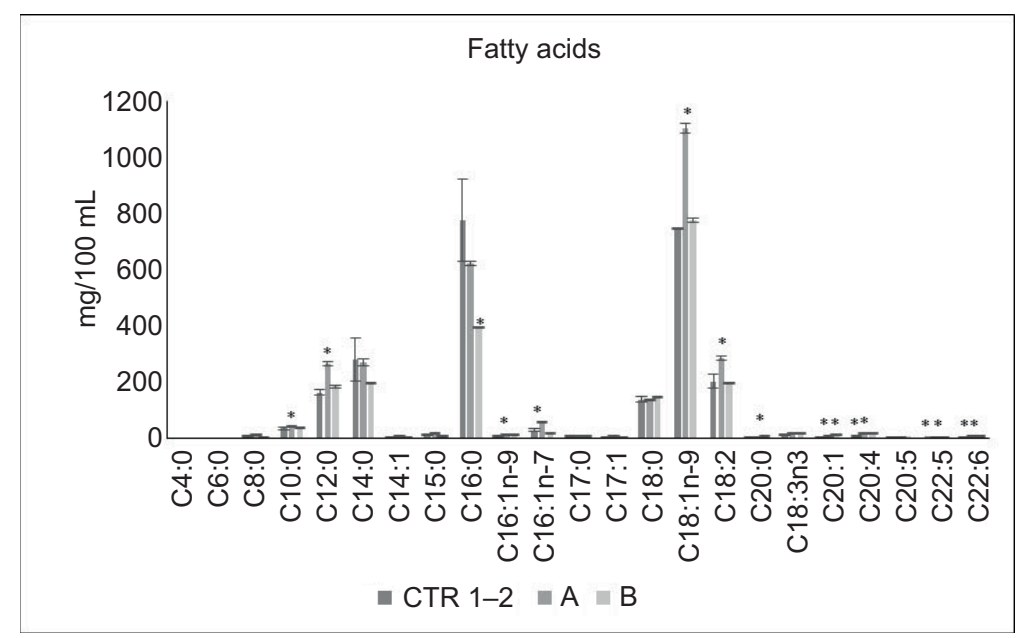

Figure 4. Fatty acid species in human breast milk. CRT: control milk samples; $A$ and B: black milk samples. Data are expressed as mean \pm SD calculated as reported in 'Statistical analysis'. Significance of A and B versus CTR $1-2 ;{ }^{*} p<0.05$.

(A)

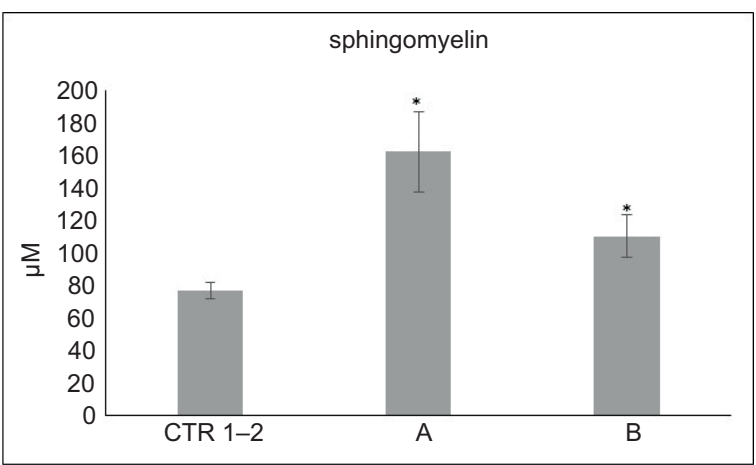

(B)

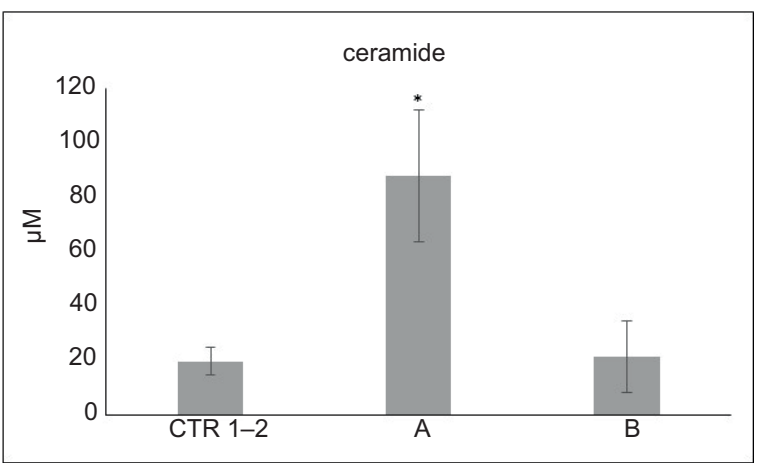

Figure 5. Total sphingomyelins and ceramides in human breast milk. CRT: control milk samples; A and B: black milk samples. Data are expressed as mean \pm SD calculated as reported in 'Statistical analysis'. Significance of A and B versus CTR 1-2; ${ }^{*} p<0.05$.

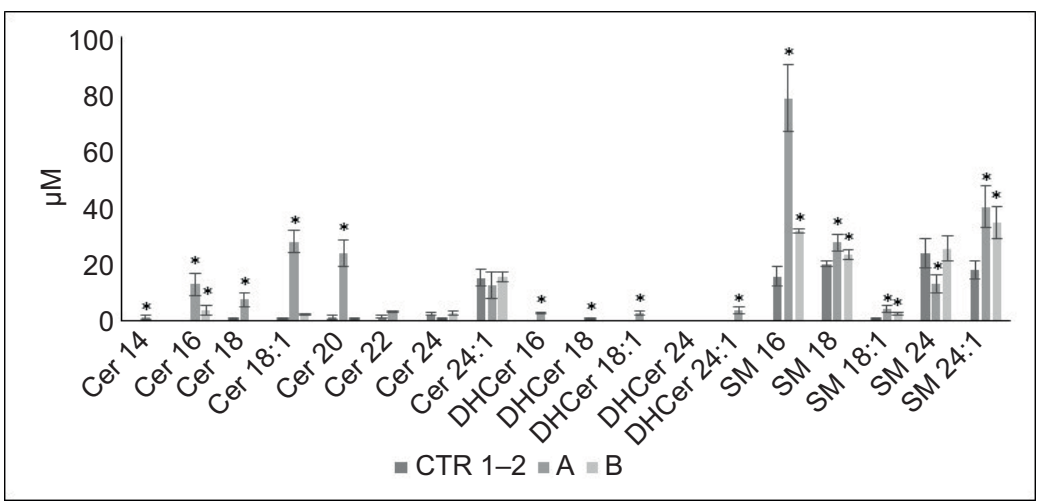

Figure 6. Species of sphingomyelins, ceramides and dihydroceramides in human breast milk. CRT: control milk samples; A and B: black milk samples. Data are expressed as mean \pm SD calculated as reported in 'Statistical analysis'. Significance of $A$ and B versus CTR 1-2; ${ }^{*} p<0.05$. 
it could just be an incidental finding of dark color HBM reported by both mothers A and B. As a matter of fact, these are the only two cases of black color HBM among a large number of pregnant/brestfeeding mothers taking this mineral globally. On discontinuation of iron intake and breastfeeding, milk in both cases again became white after a few days and both mothers restarted breastfeeding without any incident. In our experience, dark color of breast milk is not an absolute indication for discontinuation of breastfeeding.

Microbiology was aspecific. Staining with Sudan III, specific for lipids, revealed the presence of dark formations inside fat granules, which were absent in CRT milk samples. It was difficult to exactly articulate these formations, but the possibility that they were altered lipids cannot be excluded. We assumed that these could be partially oxidized lipids that precipitated as lipofuscins, which stained with Sudan III (Marani and Lazarov, 2017).

The analysis of antioxidant properties revealed that dark milk samples had a much higher antioxidant power compared to CRT milk samples. For analysis, we have chosen ORAC method was chosen because it is a robust and reliable method. In fact, the ORAC assay, other common measures of antioxidant capacity include ferric ion reducing antioxidant power and trolox equivalence antioxidant capacity assays and therefore is considered to be a preferable method because of its biologic relevance (Codini et al., 2015). A high antioxidant power is generally considered positive for the quality of a food. However, if a food has very high antioxidant properties, it becomes pro-oxidant (Mendes-da-Silva et al., 2014). In addition, it has been demonstrated that in a large number of women milk has medium level of antioxidant properties (Codini et al., 2020), similar to that we observed in two CRT milk samples. In addition, CRT milk samples had a higher sFA content than uFA, as demonstrated by Codini et al. (2020). This is a characteristic of HBM that differentiates it from formula milk, which is particularly enriched in polyunsaturated fatty acids (Codini et al., 2020). We assumed that a higher value of uFA in black milk samples predisposed them to easier oxidation.

We demonstrated that HBM of mothers who followed a Mediterranean diet had richer sphingomyelin contents than found in infant formulas (Dei Cas et al., 2021). In the present case also, we discovered similar sphingomyelin content in CRT milk samples as reported by Dei Cas et al. (2021). However, in dark milk samples, higher content values of sphingomyelin, dihydroceramide and ceramide were evident, compared to CRT milk samples. The composition between the two dark milks is different indicating a high variability in the samples. At the same time, it was possible to hypothesize that sphingolipid metabolism was altered in dark milk samples.

\section{Conclusions}

To the best of our knowledge, we reported the first two cases of black/dark gray color breast milk of women not related to minocycline therapy but having iron supplementation in common. Iron intake does not seem responsible for black milk pigmentation. The dark color of milk could be due to an alteration in antioxidant properties that become pro-oxidant. This induces a biochemical modification in lipid components in terms of fatty acids and sphingolipids. However, it was not possible to determine whether this was due to a change in the metabolic activity of the mammary gland. In summary, our data highlights that it is possible to have black/dark color milk from a healthy mother having a normal diet, and this finding does not represent an absolute indication for discontinuation of breastfeeding.

\section{References}

Ayuzo del Valle C. and Treviño Salinas E. 2014. Pink breast milk: Serratia marcescens colonization. AJP Rep. 4(2): e101e104. https://doi.org/10.1055/s-0034-1387934.

Birkholz T., Eckardt G., Renner S., Irouschek A. and Schmidt J. 2009. Green breast milk after propofol administration. Anesthesiology. 111(5): 1168-1169. https://doi.org/10.1097/ ALN.0b013e3181bbc4b1

Basler R.S. and Lynch P.J. 1985. Black galactorrhea as a consequence of minocycline and phenothiazine therapy. Case Rep Arch Dermatol. 121(3): 417-478. https://doi.org/10.1001/ archderm.1985.01660030139039

Castellote C., Casillas R., Ramirez-Santana C., Perez-Cano F.J., Castell M., Moretones M.G., et al. 2011. Premature delivery influences the immunological composition of colostrum and transitional and mature human milk. J Nutr. 141(6): 1181-1187. https://doi.org/10.3945/jn.110.133652

Ceccarini M.R., Codini M., Cataldi S., Vannini S., Lazzarini A., Floridi A., et al. 2016. Acid sphingomyelinase as target of Lycium Chinense: promising new action for cell health. Lipids Health Dis. 15(1): 183. https://doi.org/10.1186/s12944-016-0351-z

Codini M., Cataldi S., Ambesi-Impiombato F.S., Lazzarini A., Floridi A., Lazzarini R., et al. 2015. Gentamicin arrests cancer cell growth: the intriguing involvement of nuclear sphingomyelin metabolism. E Int J Mol Sci. 16: 2307-2319. https://doi. org/10.3390/ijms16022307

Codini M., Tringaniello C., Cossignani L., Boccuto A., Mirarchi A., Cerquiglini L., et al. 2020. Relationship between fatty acids composition/antioxidant potential of breast milk and maternal diet: comparison with infant formulas. Molecules. 25: E2910. https:// doi.org/10.3390/molecules25122910

Dei Cas M., Paroni R., Signorelli P., Mirarchi A., Cerquiglini L., Troiani S., et al. 2020. Human breast milk as source of sphingo lipids for newborns: comparison with infant formulas and commercial cow's milk. J Transl Med. 18: 481-494. https://doi. org/10.1186/s12967-020-02641-0 
Hanson L.A. and Korotkova M. 2002. The role of breastfeeding in prevention of neonatal infection. Semin Neonat. 7(4): 275-281. https://doi.org/10.1053/siny.2002.0124.

Hunt M.J., Salisbury E.L., Grace J. and Armati R.1996. Black breast milk due to minocycline therapy. Br J Dermatol. 134(5): 943944. https://doi.org/10.1111/j.1365-2133.1996.tb06332.x

Jenness R. 1979. The composition of human milk. Semin Perinatol. 3(3): 225-239.

Jiang C., Cheong L.Z., Zhang X., Ali A.H., Jin Q., Wei W., et al. 2021. Dietary sphingomyelin metabolism and roles in gut health and cognitive development. Adv Nutr. nmab117. https://doi. org/10.1093/advances/nmab117

Jones J., Crete J. and Neumeier R. 2014. A case report of pink breast milk. J Obstet Gynecol Neonatal Nurs. 43(5): 625-630. https:// doi.org/10.1111/1552-6909.12492

Lawrence R.A. 1985. Black galactorrhea as a consequence of minocycline and phenothiazine therapy. Case Rep Arch Dermatol. 121(3): 417-418. https://doi.org/10.1001/archderm.121.3.417

Marani E. and Lazarov N. 2017. Lipofuscin an lipofuscinosis. In: Reference Module in Neuroscience and Biobehavioral Psychology. Elsevier, Cambridge, MA. https://doi.org/10.1016/ B978-0-12-809324-5.02594-3

Mendes-da-Silva R.F., Lopes-de-Morais A.A., Bandim-daSilva M.E., Cavalcanti G.A., Rodrigues, A.R., Andrade-da-Costa B.L., et al. 2014. Prooxidant versus antioxidant brain action of ascorbic acid in well-nourished and malnourished rats as a function of dose: a cortical spreading depression and malondialdehyde analysis. Neuropharmacology. 86: 155-160. https://doi.org/10.1016/j. neuropharm.2014.06.027

Naor N., Fridman E., Kouadio F., Merlob P. and Linder N. 2019. green breast milk following ingestion of blue-green algae: a case report. Breastfeed Med. 14(3): 203-204. https://doi.org/10.1089/ bfm.2018.0184

Norris G.H., Milard M., Michalski M.C. and Blesso C.N. 2019. Protective properties of milk sphingomyelin against dysfunctional lipid metabolism, gut dysbiosis, and infammation. J Nutr Biochem. 73: 108224. https://doi.org/10.1016/j. jnutbio.2019.108224

Patton S., Canfield L.M., Huston G.E., Ferris A.M. and Jensen R.G. 1990. Carotenoids of human colostrum. Lipids. 25(3): 159-165. https://doi.org/10.1007/BF02544331

Quinn L., Ailsworth M., Matthews E., Kellams A. and Shirley DA. 2018. Serratia marcescens colonization causing pink breast milk and pink diapers: a case report and literature review. Breastfeed Med. 13(5): 388-394. https://doi.org/10.1089/bfm.2018.0002

Rainone A., Delucilla L., Elofer S., Bensimon L. and Abittan G. 2018. Propofol-induced green breast milk: a case report. Can J Hosp Pharm. 71(6): 389-391. https://doi.org/10.4212/cjhp.v71i6.2856

Riordan J. and Wambach K. 2014. Breastfeeding and Human Lactation, 4th ed. Jones and Bartlett Learning, Burlington, MA.

Skinner AM and Narchi H. 2021. Preterm nutrition and neurodevelopmental outcomes. World J Methodol. 20;11(6): 278-293. https://doi.org/10.5662/wjm.v11.i6.278

Tripaldi C., Palocci G., Di Giovanni S., Iacurto M., Steri R., Campagna M.C., et al. 2021. Effects of the drying method for flowers of Cynara cardunculus var. Altilis on milk coagulating properties. Ital J Food Sci. 33(4): 57-66. https://doi. org/10.15586/ijfs.v33i4.2026

Yazgan H., Demirdöven M., Yazgan Z., Toraman A.R. and Gürel A. 2012. A mother with green breast milk due to multivitamin and mineral intake: a case report. Breastfeed Med. 7: 310-312. https://doi.org/10.1089/bfm.2011.0048 